\title{
Biliary papillomatosis with malignant transformation: A case report and review of the literature
}

\author{
CHICHANG GU ${ }^{1,2}$, YE LIN ${ }^{2}$, HAOSHENG JIN ${ }^{2}$ and ZHIXIANG JIAN ${ }^{2}$ \\ ${ }^{1}$ Second Department of General Surgery, Shunde First People's Hospital Affiliated to Southern Medical University, \\ Foshan, Guangdong 528300; ${ }^{2}$ Department of General Surgery, Guangdong General Hospital, \\ Guangdong Academy of Medical Sciences, Guaugzhou, Guangdong 510080, P.R. China
}

Received October 10, 2014; Accepted August 17, 2015

DOI: $10.3892 / 01.2015 .3682$

\begin{abstract}
Biliary papillomatosis is a rare benign tumor that possesses a high potential for malignant transformation, and is frequently misdiagnosed. In the present report, a case of biliary papillomatosis with malignant transformation in a 63-year-old female, with a 19-year history of cholelithiasis, is presented. The patient presented with right epigastric pain and magnetic resonance imaging revealed a diffuse mass in the right liver lobe, which extended through the common bile duct. Definitive preoperative diagnosis was unable to be established; however, following surgical resection of the tumor, a diagnosis of biliary papillomatosis with malignant transformation was confirmed by pathological examination. The patient was followed up seven months later and no recurrence was detected. A supplementary review of previously published literature is additionally presented.
\end{abstract}

\section{Introduction}

Biliary papillomatosis, or intraductal papillary neoplasm of the bile duct, is a rare disease of the biliary tract characterized by the distinctive papillary proliferation of bile duct epithelial cells (1). The pathogenesis of biliary papillomatosis remains to be elucidated, however bile stasis and recurrent infection induced by hepatolithiasis or clonorchiasis likely contribute to the chronic inflammation and subsequent mucosal changes that lead to biliary papillomatosis (2). Although it is histologically categorized as benign, biliary papillomatosis should be considered to be a premalignant lesion, due to the fact that malignant transformation has been observed in $35 \%$ of cases at the time of presentation or at subsequent follow-up (3). As biliary papillomatosis is

Correspondence to: Professor Zhixiang Jian, Department of General Surgery, Guangdong General Hospital, Guangdong Academy of Medical Sciences, 106 Zhongshan Er Road, Guaugzhou, Guangdong 510080, P.R. China

E-mail:jzx_118@163.com; guchichang@126.com

Key words: biliary papillomatosis, intraductal papillary neoplasm of the bile duct, malignant, tumor a relatively rare tumor, initial and differential diagnosis is complex, and management of the tumor is typically achieved by surgical resection. In the current case report, a single case of biliary papillomatosis with malignant transformation is presented. Informed consent was obtained from the patient.

\section{Case report}

A 63-year-old female presented with right epigastric pain, and was diagnosed with cholelithiasis at the People's Hospital of Gaozhou (Maoming, China) on October 5, 2013 due to existing medical history. The patient additionally complained of fatigue, however did not exhibit any loss of weight or appetite. Previously, imaging in another hospital had revealed a mass located in the intra- and extrahepatic biliary tree, with diffuse bile duct dilation and biliary sludge. The patient was admitted to the Department of General Surgery, Guangdong General Hospital (Guangzhou, China) for further examination and treatment on October 12, 2013. During the previous 19 years, the patient had undergone three associated surgeries in other hospitals: In 1995, the patient underwent cholecystectomy due to cholecystolithiasis. In 2002, a choledocholithotomy was performed for cholangiolithiasis, and in 2007, another choledocholithotomy was performed to treat recurring cholangiolithiasis. The patient did not exhibit any respiratory, cardiovascular or constitutional symptoms. Physical examination revealed normal vital signs and mild right epigastric tenderness to palpation.

Hematological examination revealed that routine blood test results were normal. Liver function tests revealed elevated $\gamma$-glutamyl transferase (101 U/1; normal range, <32) and slightly elevated alkaline phosphatase (170 U/l; normal range, $<150$ ). Aspartate aminotransferase, alanine aminotransferase and bilirubin levels were normal. Hypokalemia was observed in the patient. With regard to tumor markers, carcinoembryonic antigen was $7.24 \mathrm{ng} / \mathrm{ml}$ (normal range, $<5$ ), and the serum levels of certain tumor markers, including $\alpha$-fetoprotein, carbohydrate antigen 19-9 and cancer antigen 125, were observed to be normal.

Abdominal ultrasonography revealed a hyperechoic mass, with an irregular surface and uneven boundary, located in the liver. Magnetic resonance imaging (MRI) and magnetic resonance cholangiopancreatography demonstrated distinct 
A

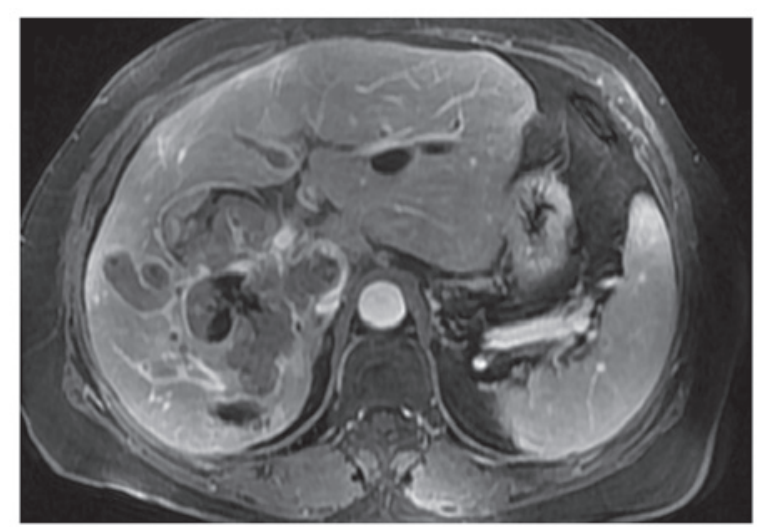

B

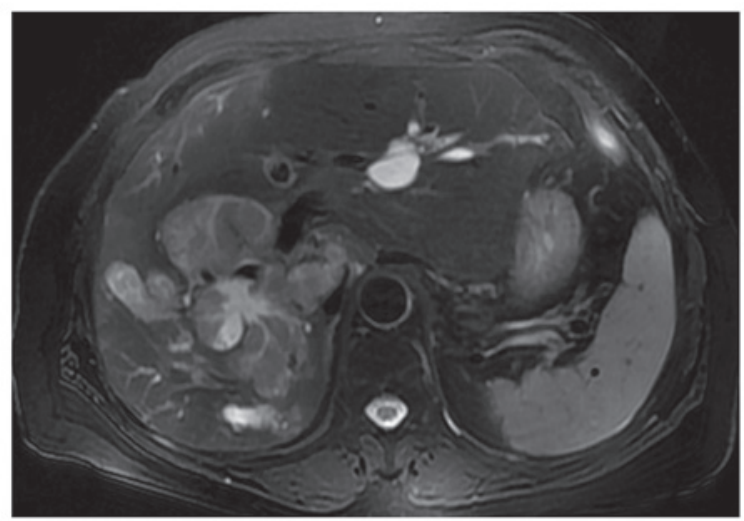

Figure 1. MRI demonstrates a diffuse and complex solid neoplasm, extending from the right intrahepatic bile duct into the common bile duct. (A) Contrast T1-weighted MRI revealed a hypointense lesion, and (B) contrast T2-weighted MRI revealed a hyperintense lesion. MRI, magnetic resonance imaging.

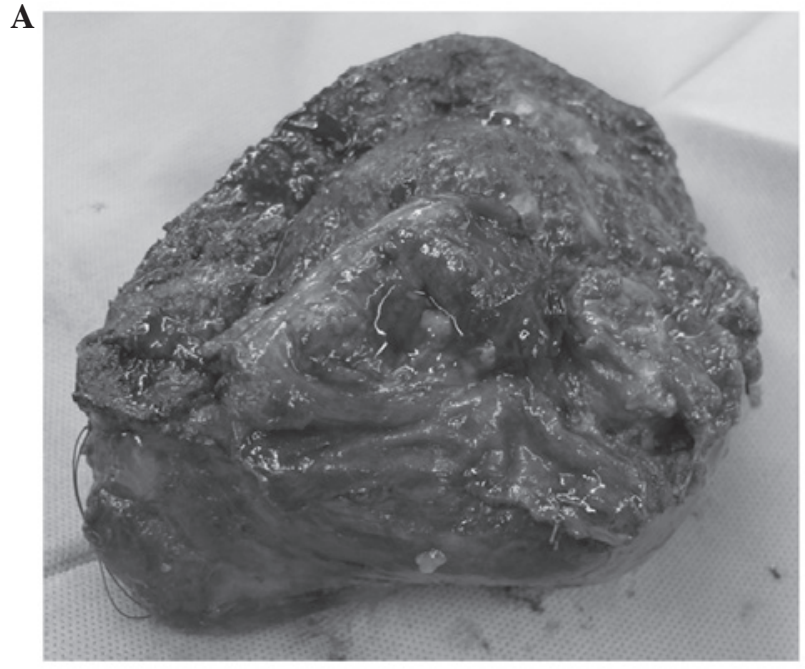

B

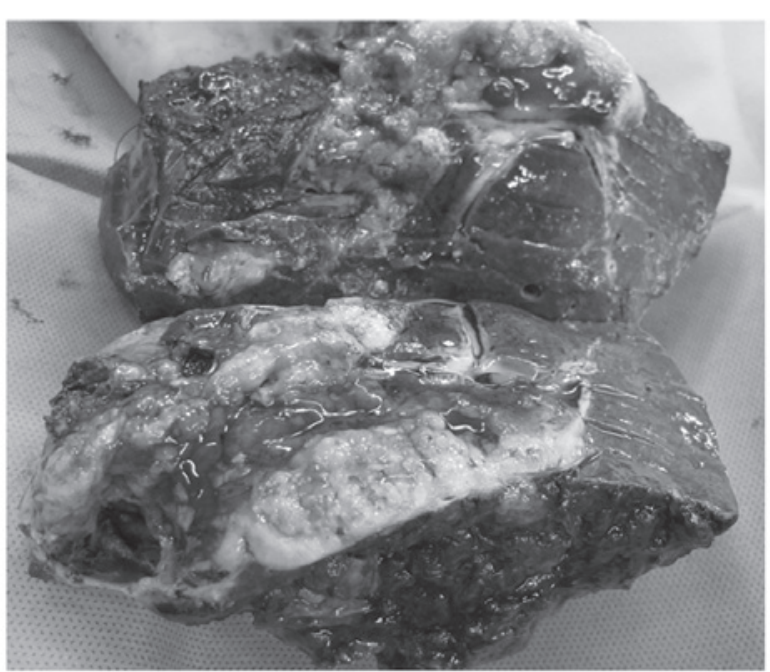

Figure 2. Evaluation of the resected tumor. (A) The resected specimen comprised the right lobe of the liver and measured $15 \times 13 \times 7 \mathrm{~cm}$. The bile ducts were distinctly dilated. (B) The common hepatic duct and bile ducts of the liver were encased by a diffuse villous tumor.

intra- and extrahepatic bile duct dilation and a T1 hypointense, T2 hyperintense, diffuse and complex solid neoplasm, extending from the right intrahepatic bile duct into the common bile duct (Fig. 1).

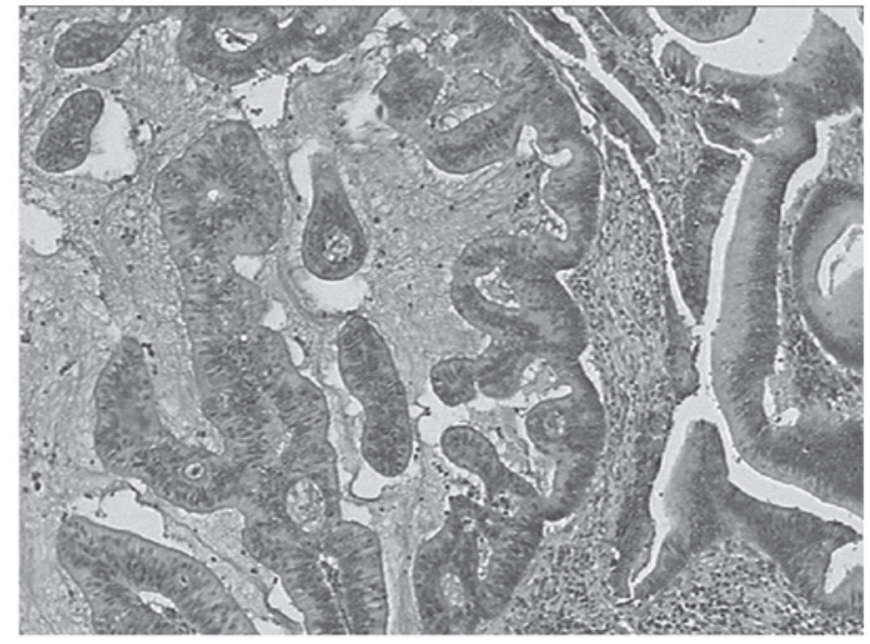

Figure 3. Microscopically, tumor cells were observed to be papillary hyperplasia, possessing cellular characteristics of high-grade dysplasia and marked nuclear pleomorphism (magnification, x400).

Preoperative diagnosis of the patient was not able to be established, however biliary papillomatosis and cholangiocarcinoma were considered during the differential diagnosis.

Surgical resection was performed on October 23, 2013. A right hepatectomy was performed by means of half liver vascular occlusion. The extrahepatic bile ducts were removed and the branch of the left hepatic duct was attached to a jejuna loop in a Roux-en-Y anastomosis. The resected specimen consisted of liver segments V-VIII and extrahepatic bile ducts. During gross inspection, the common hepatic duct appeared dilated and was occluded with a $35 \times 25 \times 15 \mathrm{~mm}$ pedunculated polypoid mass. In addition, the bile ducts of the liver were encased by a diffuse villous tumor (Fig. 2). During microscopic examination, papillary hyperplasia with the cellular characteristics of high-grade dysplasia and marked nuclear pleomorphism was observed in the dilated intra- and extrahepatic bile ducts (Fig. 3). Thus, a diagnosis of biliary papillomatosis with malignant transformation was confirmed. Following surgical resection of the tumor, the patient was followed up at seven months post surgery and no recurrence was reported. 


\section{Discussion}

Biliary papillomatosis was initially described by Chappet in 1894 (4), and 200 cases have since been described (5). Biliary papillomatosis characterized by intraductal papillary growth of biliary epithelia has been sporadically reported. This is a rare papillary or villous tumor that extends into the intraand/or extrahepatic biliary tree (2). Biliary papillomatosis may demonstrate dysplastic changes, as well as progression to carcinoma in situ and invasive adenocarcinoma (6). In addition, biliary papillomatosis is classified as one of two types, mucin-hypersecreting or non mucin-producing, depending on the presence or absence of mucin hypersecretion (7).

Biliary papillomatosis is most frequently observed in middle-aged and elderly patients, possessing a male-to-female ratio of 2:1 (7). The primary clinical symptoms of biliary papillomatosis are obstructive jaundice, repeated episodes of abdominal pain and cholangitis $(2,7,8)$. In the present case, the patient presented with right epigastric pain without jaundice and cholangitis. When the bile duct is not completely obstructed by the tumor, the above significant principal clinical symptoms may not be observed. Therefore, biliary papillomatosis may also exist without inducing significant symptoms.

In addition, laboratory findings did not aid preoperative diagnosis, owing to the absence of specificity (8). However, imaging modalities may be capable of providing more direct information. In the present case, ultrasound detection revealed dilated bile ducts and a hyperechoic mass, which was nonspecific. In computed tomography images, dilatation of intra- and extrahepatic bile ducts, as well as hypoattenuating intraductal soft-tissue masses, may be observed prior to and following administration of intravenous contrast material (9). The lesions possess low signal intensity on T1-weighted images, appear slightly hyperintense on T2-weighted images under MRI and lack significant enhancement following gadolinium administration (10).

Endoscopic retrograde cholangiopancreaticography (ERCP) demonstrates multiple filling defects and a dilated biliary tree with serrated irregularity of the bile duct wall $(9,11)$. During ERCP, a standard bile duct biopsy and/or a brush biopsy may be performed, which may aid preoperative diagnosis. However, the availability of ERCP is restricted by the associated complications, including hemorrhage, cholangitis and pancreatitis (8).

Due to the high risk of malignant transformation and the high rate of local recurrence of biliary papillomatosis, radical excision is typically the recommended treatment. When lesions are confined to a single hepatic lobe, as in the present case, partial hepatectomy is advocated (12). For lesions dispersed over the entire surface of the liver, the sole potentially curative treatment is liver transplantation (13). Curative surgical resection has been observed to result in a 5-year survival rate of up to $81 \%$ (7). Increased length of follow-up periods may be required following surgical resection, as it is estimated that biliary papillomatosis may involve the two lobes of the liver in a third of cases (14).

In conclusion, to the best of our knowledge, only a small number of cases of biliary papillomatosis have previously been reported. The present case supports the hypothesis that the progression from benign to malignant disease may follow the papilloma-carcinoma sequence (3). Although biliary papillomatosis is a rare disease, it requires increased attention due to its high malignant potential, particularly in patients with a history of cholelithiasis.

\section{References}

1. Nakajima T, Kondo Y, Miyazaki M and Okui K: A histopathologic study of 102 cases of intrahepatic cholangiocarcinoma: Histologic classification and modes of spreading. Hum Pathol 19: 1228-1234, 1988

2. Nakanuma Y, Sasaki M, Ishikawa A, Tsui W, Chen TC and Huang SF: Biliary papillary neoplasm of the liver. Histol Histopathol 17: 851-861, 2002.

3. Holtkamp W and Reis HE: Papillomatosis of the bile ducts: Papilloma-carcinoma sequence. Am J Gastroenterol 89: 2253-2255, 1994.

4. Chappet V: Cancer epithelial primitif du canal cholédoque. Lyon Med 76: 145-157, 1894.

5. Sen I, Raju RS, Vyas FL, Eapen A and Sitaram V: Benign biliary papillomatosis in a patient with a choledochal cyst presenting as haemobilia: A case report. Ann R Coll Surg Engl 94: e20-e21, 2012.

6. Neumann RD, LiVolsi VA, Rosenthal NS, Burrell M and Ball TJ: Adenocarcinoma in biliary papillomatosis. Gastroenterology 70: 779-782, 1976.

7. Lee SS, Kim MH, Lee SK, Jang SJ, Song MH, Kim KP, Kim HJ, Seo DW, Song DE, Yu E, et al: Clinicopathologic review of 58 patients with biliary papillomatosis. Cancer 100: 783-793, 2004.

8. Jiang L, Yan LN, Jiang LS, Li FY, Ye H, Li N, Cheng NS and Zhou Y: Biliary papillomatosis: Analysis of 18 cases. Chin Med J (Engl) 121: 2610-2612, 2008.

9. Levy AD, Murakata LA, Abbott RM and Rohrmann CA Jr; Armed Forces Institute of Pathology: From the archives of the AFIP. Benign tumors and tumorlike lesions of the gallbladder and extrahepatic bile ducts: Radiologic-pathologic correlation. Radiographics 22: 387-413, 2002.

10. Hoang TV and Bluemke DA: Biliary papillomatosis: CT and MR findings. J Comput Assist Tomogr 22: 671-672, 1998.

11. Kim YS, Myung SJ, Kim SY, Kim HJ, Kim JS, Park ET, Lim BC, Seo DW, Lee SK, Kim MH, et al: Biliary papillomatosis: Clinical, cholangiographic and cholangioscopic findings. Endoscopy 30: 763-767, 1998.

12. Jiang L, Yan LN, Jiang LS, Li FY, Ye H, Li N, Cheng NS and Zhou Y: Biliary papillomatosis: Analysis of 18 cases. Chin Med J (Engl) 121: 2610-2612, 2008.

13. Imvrios G, Papanikolaou V, Lalountas M, Patsiaoura K, Giakoustidis D, Fouzas I, Anagnostara E, Antoniadis N and Takoudas D: Papillomatosis of intra- and extrahepatic biliary tree: Successful treatment with liver transplantation. Liver Transpl 13: 1045-1048, 2007.

14. Yeung YP, AhChong K, Chung CK and Chun AY: Biliary papillomatosis: Report of seven cases and review of English literature. J Hepatobiliary Pancreat Surg 10: 390-395, 2003. 